\title{
Trends in incidence of infective endocarditis at the Medical Center of Alkmaar
}

\author{
M.M.G. Krul • A.B.A. Vonk • J.H. Cornel
}

Published online: 9 September 2015

(C) The Author(s) 2015. This article is published with open access at Springerlink.com

\begin{abstract}
Introduction Infective endocarditis (IE) is a life-threatening illness with a high morbidity and mortality, and with a rise in incidence in patients with prosthetic valves and cardiac devices. Recently the Dutch guidelines of IE prophylaxis have been revised, limiting IE prophylaxis to the highestrisk population. The aim of the present study was to investigate the incidence of IE and its trend between 2008-2013 in a regional hospital in the Netherlands.

Methods This is an observational descriptive study of all patients who were admitted with IE to the Medical Center of Alkmaar (MCA) from 1 January 2008 to 31 December 2013. Results A total of 89 patients with IE, including 7 patients $(7.9 \%)$ with a cardiac device IE (CDIE), were identified. In 2008 there were 8 patients with IE, this increased to 26 patients in 2013. Patients with a prosthetic valve IE increased from $25 \%$ in 2008 to $34.6 \%$ in 2013 . This increase was not seen in patients with CDIE.

Conclusion In the MCA we have observed an increase in patients with IE since 2010. This increase was in part attributable to prosthetic valve IE. A larger observational study is needed to investigate the increase of IE in the Netherlands.
\end{abstract}

Keywords Infective endocarditis - Prosthetic valve · Cardiac device $\cdot$ Endocarditis prophylaxis

M.M.G. Krul $(\bowtie) \cdot$ J.H. Cornel

Department of Cardiology, Medical Center Alkmaar,

Wilhelminalaan 12 ,

1815 JD Alkmaar, The Netherlands

e-mail: marijekrul@hotmail.com

A.B.A. Vonk

Department of Cardiothoracic surgery, VU Medical Center, Amsterdam, The Netherlands

\section{Introduction}

Infective endocarditis (IE) is a life-threatening illness with a high morbidity and mortality, despite advances in medical, surgical and critical care interventions [1]. In the past IE was a disease that commonly affected patients with predisposing valvular abnormalities caused by rheumatic carditis [2]. This presentation is currently typically seen in developing countries. Common medical conditions that put people at risk in developed countries include prior endocarditis, prosthetic valve, valvular stenosis, ventricular septal defect, mitral valve prolapse, haemodialysis and injection drug abuse [3-5].

In the Netherlands, it has been estimated that at least 300 cases of IE a year are diagnosed [4, 6, 7]. Several studies have reported a gradual increase in the incidence of IE between 1998 and 2009 [8, 9]. The incidence of IE is currently decreasing in young patients, but increases abruptly with age $[8,10]$. IE is not a uniform disease and it is evolving with changes in its microbiological profile due to a higher incidence of healthcare-associated procedures in elderly patients [11].

IE is increasingly seen in patients with prosthetic valves and intra-cardiac devices $[8,12]$. Cardiac electronic devices, including permanent pacemakers and implantable cardioverter-defibrillators (ICD), are increasingly implanted worldwide [7, 13]. The incidence of cardiac device infective endocarditis (CDIE) increased by $210 \%$ between 1993 and $2008[8,13]$. CDIE in particular has a substantially higher mortality rate than cardiac device infection without endocarditis [14].

Effective IE prophylaxis is highly desirable for IE prevention. However, there has not been a single randomised trial performed showing clear benefit of IE prophylaxis. The American Heart Association (AHA) has published recom- 


\title{
Advertisement placed here.
}

\author{
cCS Bohn \\ Stateu \\ van Loghum \\ Springer Media
}

Houten 2015 


\title{
Advertisement placed here.
}

\author{
cCS Bohn \\ Stateu \\ van Loghum \\ Springer Media
}

Houten 2015 
mendations for IE prophylaxis since 1955. In the last decade IE prevention guidelines have been changed substantially. In 2007 the AHA no longer recommended antibiotics for IE prevention before invasive gastrointestinal and genitourinary procedures [15]. Since the publication of the revised ESC guidelines also Dutch physicians changed their guidelines and limited the indication for prophylactic antibiotics to patients at the highest risk of endocarditis e.g. with a history of endocarditis, after valve replacement and patients with certain forms of congenital heart disease [16]. We have the impression that there is an increase in the incidence of IE whereas our referral population remained unchanged.

The aim of the study was to investigate if there was an increase in incidence of IE in the Medical Center of Alkmaar (MCA) between 2008 and 2013. And if so, what the explanation for this could be.

\section{Methods}

\section{Study design}

This study is an observational descriptive study of patients who were admitted with IE to the MCA from 1 January 2008 to 31 December 2013, identified by using hospital discharge records. Patient data were drawn from the database of the Diagnosis Treatment Code (DBC) case-mix system, a Diagnosis Related Group (DRG)-like system in which the resource use of all hospitalisations in the Netherlands is recorded. We considered a patient to have IE if his/her record included the DBC 702. Patients with DBC 702 were screened if they met the criteria of IE. All patients of 18 years or older with definite or possible IE, as defined by the modified Duke criteria [17], or meeting criteria for CDIE were selected. CDIE was clinically defined as clinical suspicion combined with valvular and/or lead vegetation detected by echocardiography for IE. Patients were excluded if information about IE was missing or the diagnosis was not fully proven through the Duke criteria.

Clinical characteristics including demographics, comorbid conditions, preexisting valvular conditions, and details regarding the current episode of IE (including microbiology, management, and outcome) were collected. Preexisting valvular conditions were divided into two subgroups: a high-risk group of IE defined as those with prior IE, prosthetic valve replacement or valve repair with prosthetic material, untreated or partially repaired cyanotic congenital heart disease and a moderate-risk group defined as those with prior rheumatic fever, heart murmur of evidence of native valve disease.

CDIE was scored as procedure-related IE if valvular or lead vegetation occurred $<6$ months of implantation. IE associated with valvular or lead infection occurring $>6$ months after implantation was scored as a late IE.
A prosthetic valve endocarditis was classified as procedure related if valvular vegetation occurred $<1$ year after implantation of the prosthetic valve. Prosthetic valve endocarditis was defined as late IE if IE occurred $>1$ year after implantation [11].

Due to the retrospective nature of the study, approval of the local ethics committee was not necessary.

\section{Statistical analysis}

Continuous variables are presented as median and interquartile range (IQR) and categorical variables are presented as frequencies with percentages. One-sample KolmogorovSmirnov test were used to test continuous data for normal distribution. Pearson Chi-square tests were used to test dichotomous parameters. $P$-values $<0.05$ were considered significant. Statistical Package for Social Sciences V.20 (SPSS Inc., Chicago IL, USA) was used.

\section{Results}

Study population

A total of 91 patients were documented with IE, of which two were excluded from the current analysis because of missing information. The 89 patients with IE included 7 patients $(7.9 \%)$ with CDIE.

The clinical characteristics of IE are shown in Table 1. Patients were predominantly men $(69.7 \%)$, with a median (IQR) age of 68 (59.2-75.4) years. Blood cultures were positive in 82 patients $(92.1 \%)$. Staphylococcus aureus was most frequently found in the isolates $(24.4 \%)$. Also Streptococcus oralis (11\%), Streptococcus bovis (9.8\%) and Enterococcus faecalis ( $8.5 \%$ ) were frequently seen. IE prophylaxis was indicated in 21 high-risk patients. All 21 patients were treated according the Dutch guidelines.

Sixteen patients (18\%) with IE died in hospital. In medically treated patients the mortality rate was $22.6 \%$ (12 out of 53 ) whereas 4 out of $36(11.1 \%)$ patients died after valve replacement or device removal $(p=0.164)$.

\section{Valve infections}

A valve IE was present in 82 patients $(92.1 \%)$. IE involved a native valve in 63 patients $(70.8 \%)$ and a prosthetic valve in 19 patients $(21.3 \%)$. The distribution of native valve involvement includes the aortic $(n=26)$, mitral $(n=29)$, pulmonary $(n=2)$ and both aortic and mitral $(n=6)$ valves. Five patients $(7.9 \%)$ with a native valve had a severe aortic valve stenosis, four $(6.3 \%)$ patients had a bicuspid aortic valve and two $(3.2 \%)$ had a mitral valve prolapse. The distribution 
Table 1 Demographic characteristics of patients with infective endocarditis between 2008-2013

\begin{tabular}{ll}
\hline Characteristic & $N=89(\%)$ \\
\hline Male gender & $62(69.7)$ \\
Age (years) & $68(59.2-75.4)$ \\
Native valve & $63(70.8)$ \\
Prosthetic valve & $19(21.3)$ \\
CDIE & $7(7.9)$ \\
Positive blood culture & $82(92.1)$ \\
Staphylococcus aureus & $20(24.4)$ \\
Streptococcus oralis & $9(11)$ \\
Streptococcus bovis & $8(9.8)$ \\
Enterococcus feacalis & $7(8.5)$ \\
Mortality & $16(18)$ \\
\hline
\end{tabular}

Categorical variables are expressed as count (percentage). Continuous variables are expressed as median (IQR).

CDIE cardiac device infective endocarditis.

of prosthetic valve involvement includes the aortic $(n=17)$, mitral $(n=3)$ and both aortic and mitral $(n=1)$ valves.

All patients received high-dose antibiotics intravenously. Valve replacement was needed in $41.5 \%$ patients.

In $31.6 \%$ of the patients with a prosthetic valve a procedure-related infection was present. The median time of an early prosthetic valve IE was 2.0 (IQR $0-4.75$ ) months.

\section{Lead infections}

CDIE was present in 7 patients (7.9\%). Coexisting valve infection in patients with CDIE was not found. All patients with CDIE had a lead infection. Six patients with CDIE had an ICD, and 1 patient a DDDR pacemaker.

All patients received high-dose antibiotics intravenously. Device and lead removal was performed in 2 of 7 patients (28.6\%), both patients had an early lead infection. Device and lead removal was performed in both patients because of growth of vegetation, septic emboli to lung and spondylitis. The median time of an early CDIE was 3.5 (IQR 3.0-3.5) months.

\section{Ie 2008-2013}

The number of patients with IE between 2008 and 2013 is shown in Fig. 1. We observed an increase in incidence of IE especially in the last 3 years. This increase is in part attributable to patients with a prosthetic valve. A prosthetic valve infection occurred in almost $25 \%$ of IE cases per year and increased from $25 \%$ ( 2 out of 8 ) patients in 2008-38.9\% (7 out of 18) in 2012. The number of patients with CDIE in the years between 2008 and 2013 is also displayed in Fig. 1. The number of patients with CDIE is limited.

Table 2 displays age and predisposing factors for IE. As shown in Table 2, there was no increase in age of IE patients between 2008 and 2013. The contribution of patients with predisposing conditions increased especially in patients

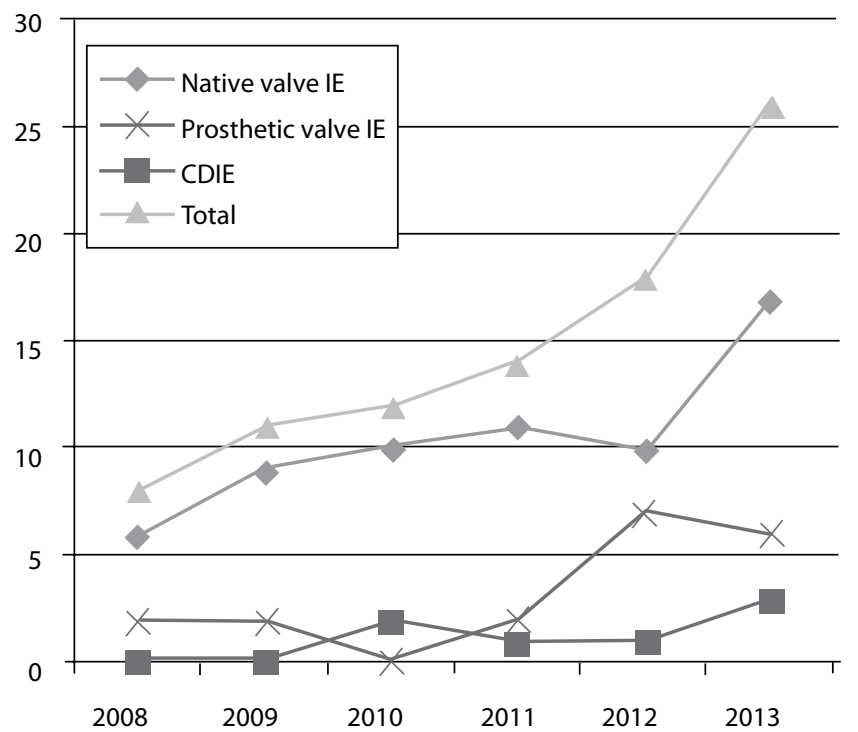

Fig. 1 Number of patients with infective endocarditis, prosthetic valve endocarditis and CDIE between 2008-2013

with high-risk IE. As mentioned before, this increase was in part attributable to prosthetic valve IE.

\section{Discussion}

In the MCA we have observed an increase in patients with IE over the last 6 years, especially in the last 3 years. The increase of IE in patients with predisposing conditions was especially clear in the high-risk group, whereas IE in the moderate-risk group remained stable. This increase was largely attributable to prosthetic valve endocarditis, but not to CDIE.

\section{Prophylaxis}

Effective prophylaxis is highly desirable for IE prevention. The last decade IE prevention guidelines have been changed substantially. In 2007, the AHA no longer recommended antibiotics for IE prevention before invasive gastrointestinal and genitourinary procedures [15]. After publication of these guidelines, in the first 2 years Thornhill et al. showed a $78.6 \%$ reduction in the prescription of antibiotic prophylaxis, yet they did not detect a significant increase in the number of IE cases above the long-term baseline trend during their study of the 2007 AHA endocarditis prevention guidelines [18-21]. Since the publication of the revised ESC guidelines, also Dutch physicians changed their guidelines and limited the indication for prophylactic antibiotics to patients at highest risk of endocarditis [16].

Several studies have not shown an increase in the incidence of IE after implementation of the AHA guidelines 
Table 2 Comorbidities and predisposing factors among cases of infective endocarditis between 2008-2013

\begin{tabular}{|c|c|c|c|c|c|c|}
\hline & Age (years) & $\begin{array}{l}\text { Prosthetic valve endocarditis or CDIE } \\
N(\%)\end{array}$ & $\begin{array}{l}\text { Prior IE } \\
N(\%)\end{array}$ & $\begin{array}{l}\text { High risk } \\
N(\%)\end{array}$ & $\begin{array}{l}\text { Moderate risk } \\
N(\%)\end{array}$ & $\begin{array}{l}\text { Total amount of IE } \\
N\end{array}$ \\
\hline 2008 & $69.1(66.1-76.8)$ & $2(25)$ & $0(0)$ & $2(25)$ & $1(12.5)$ & 8 \\
\hline 2009 & $67.2(63.9-79.8)$ & $3(27.3)$ & $0(0)$ & $2(18)$ & $2(18)$ & 11 \\
\hline 2010 & $68.0(51.2-76.8)$ & $3(25)$ & $0(0)$ & $3(25)$ & $4(33)$ & 12 \\
\hline 2011 & $65.5(53.1-75.3)$ & $3(21.4)$ & $1(7.1)$ & $3(21)$ & $6(42)$ & 14 \\
\hline 2012 & $73.2(64.9-76.4)$ & $9(50)$ & $1(5.5)$ & $9(50)$ & $4(22)$ & 18 \\
\hline 2013 & $68.3(53.6-75.3)$ & $9(34.6)$ & $1(3.8)$ & $10(38)$ & $1(3.8)$ & 26 \\
\hline
\end{tabular}

Categorical variables are expressed as count (percentage). Continuous variables are expressed as median (IQR)

$I E$ infective endocarditis.

High risk: prior IE, prosthetic valve or valve repair with prosthetic material, untreated or partially repaired congenital heart disease.

Moderate risk: previous rheumatic fever, heart murmur of evidence of native valve disease.

$[22,23]$. A recently published study by Dayer et al. showed that since the introduction of the 2008 NICE (National Institute for Health and Clinical Excellence) guidelines for IE prophylaxis, abolishing the need for preventive treatment, prescriptions of antibiotic prophylaxis have fallen substantially. At the same time the incidence of IE rose significantly. Per month 35 more cases of IE were reported than would have been expected [24].

Our study also shows an increase of patients with IE. In contrast to Dayer et al. we did not study the influence of the change in guidelines for IE prophylaxis but tried to unravel the reasons behind the increased incidence. Before interpreting these data, we must realise that IE is an evolving disease, with various confounders that could account for an increase of IE. It could be caused by a higher incidence of healthcare-associated cases, increased number of patients with implanted intracardiac devices, increased prevalence of diabetes mellitus, or increased chronic dialysis, and ageing population [25]. Secondly, there is a major difference between the guidelines in the United Kingdom and in the Netherlands. The NICE guidelines recommended complete cessation of IE prophylaxis, whereas the Dutch guidelines limited IE prophylaxis to patients with the highest risk of IE, e.g. with a history of IE, after valve replacement and patients with certain forms of congenital heart disease $[16,26]$.

Ongoing data monitoring and further clinical trials are needed to determine if antibiotic prophylaxis still has a role in protecting some patients at particularly high risk. For now, there is no advice to change the guidelines of IE prophylaxis.

\section{Repeat IE}

Three of our patients had a repeat of IE, as shown in Table 2. Alagna et al. showed that repeat IE was associated with haemodialysis, HIV, injection drug use, Staphylococcus aureus IE, healthcare acquisition and prior IE. Patients with repeat IE had a higher 1-year mortality than those with single episode [17]. In our study the rate of repeat IE was low (2.2\%). In a larger multicentre study the rate of repeat IE was in the same range $(4.8 \%)$. Some studies have identified prosthetic valve IE as a possible risk factor for repeat IE. Mansur et al. reported that having a prosthetic valve IE in the first year after a valve replacement is a risk factor for having additional episodes [27]. Renzulli et al. showed that prosthetic valve IE was an independent risk factor for recurrence of IE [28]. We found only 1 procedure-related prosthetic valve endocarditis.

\section{Predisposing factors for IE}

Several larger studies have shown an increase of IE in older patients [6]. Many factors are related to this shift in age distribution: (1) age of the population has been increasing steadily; (2) people with rheumatic or congenital heart disease are surviving longer; (3) more frequent prosthetic valve surgery; (4) new at-risk groups have emerged, including intravenous drug users, patients with cardiac devices, and those exposed to healthcare associated bacteraemia. Our study did not show an increase in age in patients with IE between 2008 and 2013. It is possible that the combination of more healthcare-associated procedures without IE prophylaxis and less rigid IE prophylaxis in patients with previous known valve disease contribute to the rise incidence of IE. A larger observational study is needed to investigate the increase in IE in the Netherlands.

\section{Limitations}

We describe a single-centre observation, with small numbers of IE. The population in the MCA may not truly represent the general population. Secondly, the diagnosis of underlying heart disease was obtained from medical records and was based primarily on echocardiography and physician assessment without consistent pathological confirmation. Thirdly, data on patients with pocket infections were not collected, so the relationship between pocket infection and IE and CDIE could not be evaluated rigorously. 


\section{Conclusions}

In the MCA we have observed an increase in patients with IE since 2010. This increase is in part attributable to prosthetic valve IE. A larger observational study is needed to investigate the increase of IE in the Netherlands.

\section{Funding None.}

Conflict of interests None declared.

Open Access This article is distributed under the terms of the Creative Commons Attribution License which permits any use, distribution, and reproduction in any medium, provided the original author(s) and the source are credited.

\section{References}

1. Baddour LM, Wilson WR, Bayer AS, et al. Infective endocarditis: diagnosis, antimicrobial therapy, and management of complications: a statement for healthcare professionals from the Committee on Rheumatic Fever, Endocarditis, and Kawasaki Disease, Council on Cardiovascular Disease in the Young, and the Councils on Clinical Cardiology, Stroke, and Cardiovascular Surgery and Anesthesia, American Heart Association: endorsed by the Infectious Diseases Society of America. Circulation. 2005;111:e394-434.

2. Griffin MR, Wilson WR, Edwards WD, et al. Infective endocarditis. Olmsted County, Minnesota, 1950 through 1981. JAMA. 1985;254:1199-202.

3. Mylonakis E, Calderwood SB. Infective endocarditis in adults. N Engl J Med. 2001;345:1318-30.

4. Bor DH, Woolhandler S, Nardin R, et al. Infective endocarditis in the U.S., 1998-2009: a nationwide study. PLoS One. 2013;8:e60033.

5. Murdoch DR, Corey GR, Hoen B, et al. Clinical presentation, etiology, and outcome of infective endocarditis in the 21st century: the International Collaboration on Endocarditis-Prospective Cohort Study. Arch Intern Med. 2009;169:463-73.

6. Habib G, Hoen B, Tornos P, et al. Guidelines on the prevention, diagnosis, and treatment of infective endocarditis (new version 2009): the Task Force on the Prevention, Diagnosis, and Treatment of Infective Endocarditis of the European Society of Cardiology (ESC). Endorsed by the European Society of Clinical Microbiology and Infectious Diseases (ESCMID) and the International Society of Chemotherapy (ISC) for Infection and Cancer. Eur Heart J. 2009;30:2369-413.

7. van der Meer JT, Thompson J, Valkenburg HA, et al. Epidemiology of bacterial endocarditis in The Netherlands. I. Patient characteristics. Arch Intern Med. 1992;152:1863-8.

8. Greenspon AJ, Patel JD, Lau E, et al. 16-year trends in the infection burden for pacemakers and implantable cardioverter-defibrillators in the United States 1993 to 2008. J Am Coll Cardiol. 2011;58:1001-6.

9. Stofmeel MA, Kelder JC, van Hemel NM, et al. [Implanted pacemakers in the Netherlands, 1984-1997; number, types and patient characteristics]. Ned Tijdschr Geneeskd. 2001;145:1254-8.

10. Sohail MR, Uslan DZ, Khan AH, et al. Management and outcome of permanent pacemaker and implantable cardioverter-defibrillator infections. J Am Coll Cardiol. 2007;49:1851-9.

11. Hoen B, Alla F, Selton-Suty C, et al. Changing profile of infective endocarditis: results of a 1-year survey in France. JAMA. 2002;288:75-81.
12. Cabell CH, Jollis JG, Peterson GE, et al. Changing patient characteristics and the effect on mortality in endocarditis. Arch Intern Med. 2002;162:90-4.

13. Knoll B, Baddour LM, Wilson WR. In: Mandell GL, Bennett JE, Dollin R, editors. Mandell, douglas and benett's principles and practice of infectious diseases. 2010. pp 1113-26.

14. Wang A, Athan E, Pappas PA, et al. Contemporary clinical profile and outcome of prosthetic valve endocarditis. JAMA. 2007;297:1354-61.

15. Wilson W, Taubert KA, Gewitz M, et al. Prevention of infective endocarditis: guidelines from the American Heart Association: a guideline from the American Heart Association Rheumatic Fever, Endocarditis and Kawasaki Disease Committee, Council on Cardiovascular Disease in the Young, and the Council on Clinical Cardiology, Council on Cardiovascular Surgery and Anesthesia, and the Quality of Care and Outcomes Research Interdisciplinary Working Group. J Am Dent Assoc. 2007;138:739-60.

16. van der Meer JT, van Drenth J, van den Brink RBA, et al. Preventie bacteriele endocarditis. Hartstichting 2008. https://www.nvvc. nl/media/richtlijn/49/endocarditisprofylaxefolder\%20artsen.pdf.

17. Alagna L, Park LP, Nicholson BP, et al. Repeat endocarditis: analysis of risk factors based on the International Collaboration on Endocarditis-Prospective Cohort Study. Clin Microbiol Infect. 2014;20:566-75.

18. Thornhill MH, Dayer MJ, Forde JM, et al. Impact of the NICE guideline recommending cessation of antibiotic prophylaxis for prevention of infective endocarditis: before and after study. BMJ. 2011;342:d2392.

19. HCUP databases. Overview of the nationwide inpatient sample (NIS). Healthcare Cost and Utilization Project (HCUP). 2007 2009. Rockville, MD: Agency for Healthcare Research and Quality; November 2011. www.hcup-us.ahrq.gov/nisoverview/jsp/. Accessed 1 Sept 2010.

20. Rogers AM, Schiller NB. Impact of the first nine months of revised infective endocarditis prophylaxis guidelines at a university hospital: so far so good. J Am Soc Echocardiogr. 2008;21:775.

21. Richey R, Wray D, Stokes T. Prophylaxis against infective endocarditis: summary of NICE guidance. BMJ. 2008;336:770-1.

22. DeSimone DC, Tleyjey IM, Correa DD. Incidence of infective endocarditis caused by viridans group streptococci before and after publication of the 2007 American Heart Association's endocarditis prevention guidelines. Circulation. 2012;126:60-4.

23. Bekdeli B, Wang Y, Kim N. Trends in hospitalization rates and outcomes of endocarditis among Medicare beneficiaries. J Am Coll Cardiol. 2013;62:2217-26.

24. Dayer MJ, Jones S, Prendergast B. Incidence of infective endocarditis in England, 2000-13: a secular trend, interrupted time-series analysis. Lancet. 2014. doi:10.1016/S0140-6736(14)62007-9. (pii: S0140-6736(14)62007-9).

25. Duval X, Hoen B. Prophylaxis for infective endocarditis: let's end the debate. Lancet. 2014. doi:10.1016/S0140-6736(14)62121-8. (pii: S0140-6736(14)62121-8).

26. NICE. Prophylaxis against infective endocarditis. National Institute for Health and Clinical Excellence. 2008. http://www.nice. org.uk/CG064. Accessed 31 Oct 2013.

27. Mansur AJ, Dal Bo CM, Fukushima JT, et al. Relapses, recurrences, valve replacements, and mortality during the long-term followup after infective endocarditis. Am Heart J. 2001;141:78-86.

28. Renzulli A, Carozza A, Romano G, et al. Recurrent infective endocarditis: a multivariate analysis of 21 years of experience. Ann Thorac Surg. 2001;72:39-43. 